\title{
Archéopages
}

Archéopages Archéologie et société

39 | 10/2013-01/2014

Le sort des vaincus

\section{Un service à vin en alliage cuivreux à Argentomagus}

\section{Yoann Rabasté}

\section{OpenEdition}

Journals

Édition électronique

URL : https://journals.openedition.org/archeopages/569

DOI : 10.4000/archeopages.569

ISSN : 2269-9872

\section{Éditeur}

INRAP - Institut national de recherches archéologiques préventives

\section{Édition imprimée}

Date de publication : 1 septembre 2014

Pagination : 102-103

ISSN : 1622-8545

\section{Référence électronique}

Yoann Rabasté, «Un service à vin en alliage cuivreux à Argentomagus », Archéopages [En ligne], 39 |

10/2013-01/2014, mis en ligne le 01 janvier 2016, consulté le 28 janvier 2022. URL : http:// journals.openedition.org/archeopages/569; DOI : https://doi.org/10.4000/archeopages.569 


\section{Un service à vin en alliage cuivreux à Argentomagus}

Yoann Rabasté

Inrap, responsable d'opération

Site

Argentomagus

Saint-Marcel

Indre

Date

Juillet 2013

Superficie

$230 \mathrm{~m}^{2}$

\section{Équipe}

Infographie/responsable de secteur

Cyril Van Lynden Tot Oldenaller, Association pour la Sauvegarde du Site Archéologique d'Argentomagus et Amis du Musée (ASSAAM)

Étude du mobilier

Michaël Brunet, Archéograph

Analyses chimiques

Nicolas Garnier, Laboratoire Nicolas Garnier (Lng)

Consolidation/restauration

Christiane Sire, Ministère de la Culture

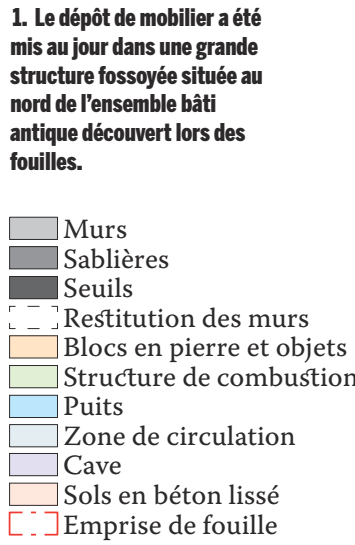

1. Le dépôt de mobilier a été Jour dans une grande nord de l'ensemble bât antique découvert lors des

Restitution des murs

Blocs en pierre et objets

Zone de circulation

Sols en béton lissé

[.] Emprise de fouille le glissement de la plateforme, à la suite de l'effondrement partiel du mur à contreforts.

Au cours de cette période, une grande structure fossoyée, de 4,20 m de long pour 1,90 m de large, est installée perpendiculairement au mur de façade nord du bâtiment [ill. 1]. La fonction de cette structure, fouillée seulement en partie pour l'instant, reste encore indéterminée, même si plusieurs indices (pierres rubéfiées, couches de charbon, etc.) laissent supposer une activité de combustion.

Un dépôt d'objets a été retrouvé dans le comblement supérieur de l'angle sud-est de la structure. Sur les quinze pièces qui le composent, cinq correspondent à des vases en céramique et dix sont en alliage cuivreux. Les pièces - à l'exception de deux vases en céramique - étaient disposées dans un grand chaudron [ill. 2]. Le site d'Argentomagus n'avait pas connu de découverte aussi importante depuis celle du Mercure en Bronze (aujourd'hui emblème du site). En outre, ce dépôt présente un grand intérêt scientifique, puisqu'il a fait l'objet d'un nouveau protocole d'extraction des composés organiques. Si des analyses sont régulièrement effectuées, il s'agit ici d'une première sur du mobilier métallique découvert in situ avant traitement.

Les vases en céramique sont typiques de ceux de l'Antiquité tardive ; quant aux objets en alliages cuivreux, ils renvoient à des formes et des décors qui courent tout au long de la période antique (Rabasté et al., 2014). Toutefois, parmi ce dépôt quatre pièces sont particulièrement remarquables du fait de leurs décors [ill. 3a].

Le pot présente une anse décorée reposant sur deux têtes d'oiseau au long bec, avec deux feuilles latérales, recourbées en volutes. La face antérieure présente un décor mouluré représentant Hercule se reposant sur sa massue, avec au-dessus deux éléments dont l'identification reste incertaine. La partie sommitale figure son fils Télèphe, nourri par une biche [ill. 3b]. La forme de ce pot appartient à un type rare en Italie, mais extrêmement répandu dans les provinces romaines. Quant au décor de l'anse, bien que certaines soient produites dans les provinces occidentales de l'Empire, peu d'exemplaires ont jusqu'ici été mis au jour. Nos premières recherches ne nous ont pas permis de trouver un exemplaire similaire.

La coupelle circulaire possède un décor qui se caractérise par un bossage sur la quasi-totalité de la superficie de la panse et sur la lèvre [ill. 3c]. Le décor se traduit par une série de gouttes ou de feuilles d'eau, surmontée par une ligne en relief, au-dessus de laquelle est placée une frise de moulures obliques, qui donne naissance à un bord plus ou moins perlé. Aucun exemplaire

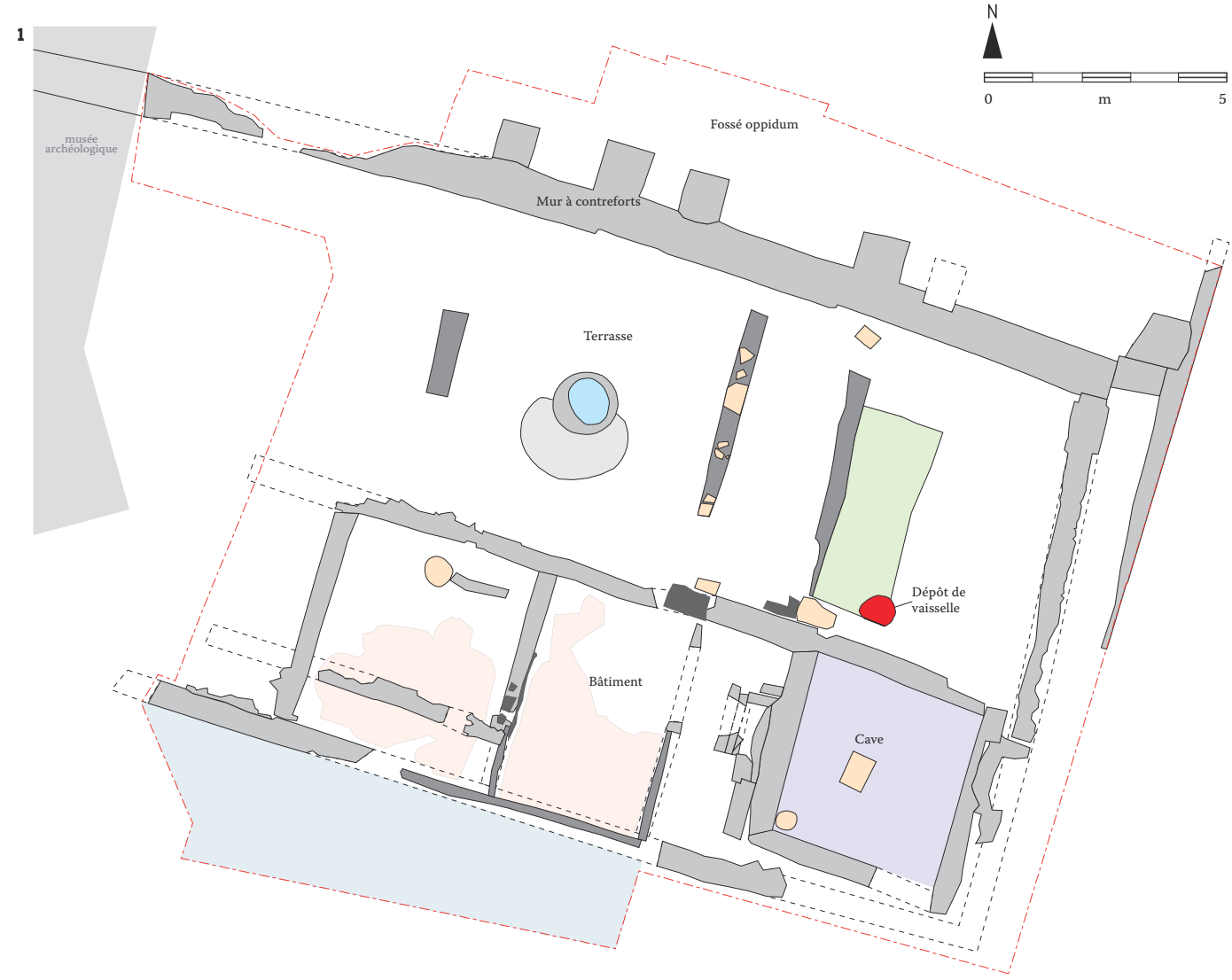





semblable n'a été découvert en l'état actuel de nos connaissances, bien que le décor de feuilles d'eau se retrouve sur quelques mobiliers métalliques.

La particularité du bassin se caractérise par la présence d'un bec verseur à tête zoomorphe, fixé contre le filtre, représentant un bovidé ou un porc [ill. 3a]. Bien que quelques exemplaires possèdent des becs verseurs zoomorphes, ce type de décors se rencontre rarement, laissant place à des becs non figuratifs ou à des décors d'animaux fantastiques. L'exemplaire le plus proche de celui-ci a été retrouvé à Verulamium (Grande-Bretagne) dans un contexte daté de 300-315 de notre ère, figurant une tête de taureau.

Étamé, le plat présente un médaillon où un griffon assis, placé quasiment de profil sur un support décoré non identifié, regarde vers l'arrière [ill. 3d]. Cet ensemble est bordé d'une ligne torsadée et de deux lignes plus larges, incisées en léger relief. En l'état actuel, aucun parallèle n'a été identifié, bien que des plats avec médaillons aient déjà été découverts.

Les formes de ces objets conduisent à reconnaître un service à vin, ce que confirment les résultats des analyses chimiques. En effet, le principal composé rencontré, l'acide tartrique, marqueur du raisin, est représenté sur la quasi-totalité des pièces. Des traces d'acide syringique, marqueur de la dégradation de la malvidine - tannin spécifique au raisin noir et teinturier et donnant la couleur au vin rouge -, ont été observées sur l'un des objets. Malgré le caractère encore partiel de l'étude, les premières comparaisons permettent de situer l'ensemble du dépôt vers le fin du III ${ }^{\mathrm{e}}$-début du $\mathrm{IV}^{\mathrm{e}}$ siècle de notre ère. Le médaillon représentant un griffon évoque cependant un style décoratif $\mathrm{du} \mathrm{IV}^{\mathrm{e}}$ siècle que l'on retrouve sur

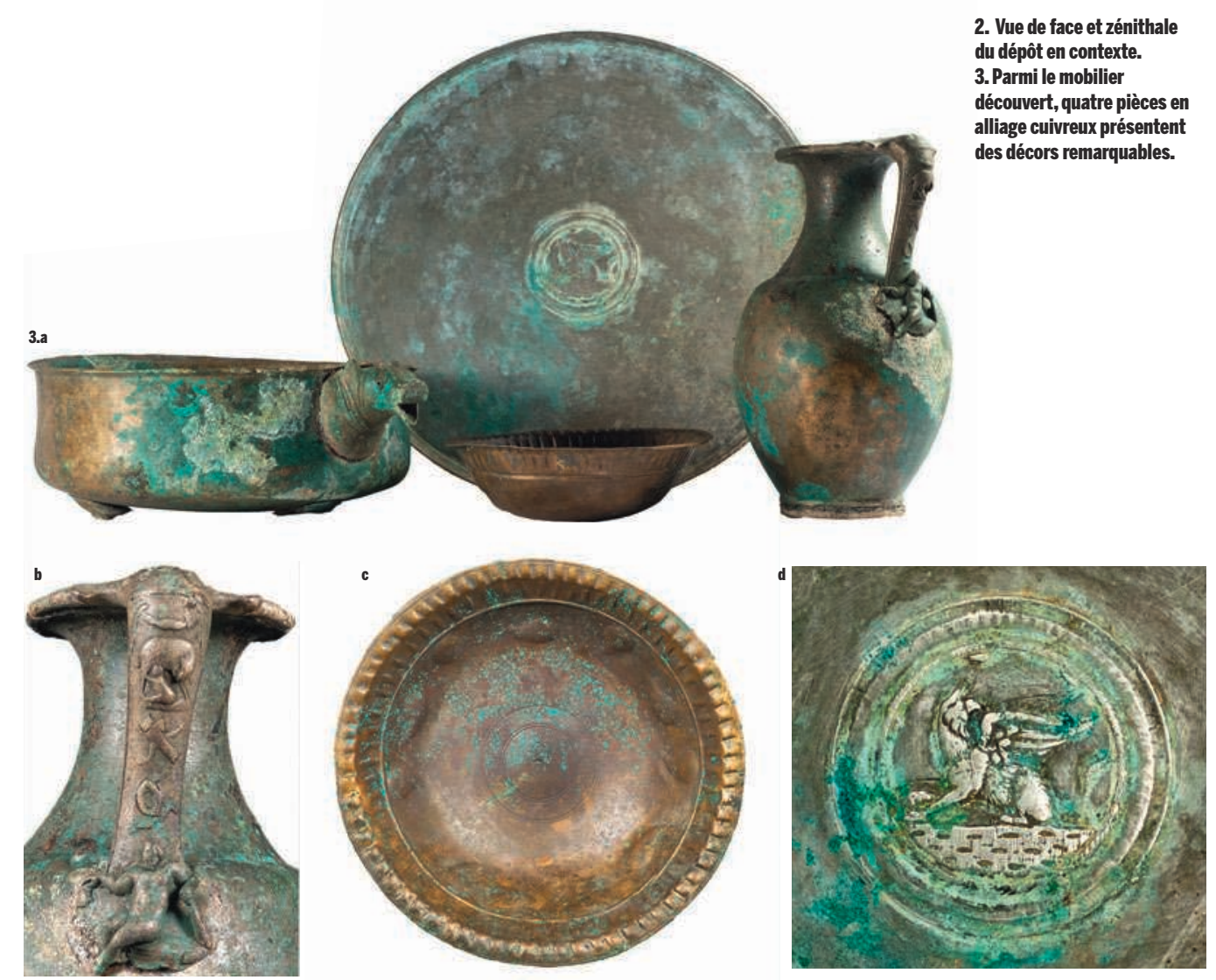

l'argenterie de Mildenhall (GrandeBretagne). Cette proposition chronologique se précise avec le mobilier issu du comblement de la structure de combustion, daté du IV ${ }^{e}$ siècle (Rabasté et al., 2014).

Le contexte de ce dépôt est encore mal connu, mais l'aspect cultuel semble exclu compte tenu de sa localisation dans la structure de combustion ; l'absence de recreusement dans le comblement exclut également l'hypothèse d'une cache. Il s'agit plus vraisemblablement d'un dépôt, effectué au moment du comblement de la structure dont l'objectif est inconnu et oublié par la suite, témoignant ainsi du statut élevé du propriétaire.
Références bibliographiques

Rabasté Y., VAn LyNden Tot Oldenaller C., Bouchain-Palleau I. et Le GofF E., 2013, Saint-Marcel (36, Indre), "Les Mersans », Poursuite de

la fouille de l'ensemble monumental aux abords immédiats est du musée archéologique d'Argentomagus, Rapport final d'opération de fouille programmée, Campagne de 2012, Assaam-Inrap-SRA Centre, 146 p.
Rabasté Y., VAN Lynden Tot OldENALler C., Bouchain-Palleau I., Boislève J., Garnier N. et Brunet M., 2014, Saint-Marcel (36, Indre), «Les Mersans », L'ensemble monumental à l'est du musée archéologique d'Argentomagus, Rapport intermédiaire de fouille programmée triennale, Campagne de 2013, Assaam-Inrap-SRA Centre, 280 p. 